E0283 RESEARCH ON THE RELATIONSHIP OF ANXIETY SYMPTOMS TO THE QUALITY OF LIFE IN PATIENTS WITH ACUTE CORONARY SYNDROME

doi:10.1136/hrt.2010.208967.283

${ }^{1}$ Liu Guanghui, ${ }^{2}$ Ma Wenlin, ${ }^{3}$ Wu Xianzheng, ${ }^{3}$ Zhu Chenting, ${ }^{3}$ Pan Juping, ${ }^{3}$ Guo Gang, ${ }^{4} \mathrm{Hu}$ Dayi. ${ }^{1}$ Emergency Internal Medicine, Tongji Hospital, Tongji University; ${ }^{2}$ Department of Cardiology, Tongji Hospital, Tongji University; ${ }^{3}$ Emergency Internal Medicine, Tongji Hospital, Tongji University; ${ }^{4}$ Heart Center Peking University Peoples Hospital

Objective Anxiety is thought to be a common symptom in patient $\mathrm{s}$ who suffer from acute coronary syndrome (ACS). This study aims to present a general data about the anxiety symptoms in Chinese ACS patients, and evaluate the impact of anxiety symptoms on their quality of life (QOL).

Method We enrolled 454 Chinese patient s with ACS from ShangHai, whose baseline data were obtained within 1 week after hospital admission. The subscale of Chinese version of the Hospital Anxiety and Depression Scale (HADS-A) and SF-36 were used to assess anxiety and their QOL, respectively. All the participant s were divided into 2 main groups: $\mathrm{HADa} \geq 6(\mathrm{n}=247)$ and $\mathrm{HADa} 0.05)$. The participants from non-anxiety group had a better QOL than anxiety group $(p<0.01)$. And anxiety was negatively associated with the QOL $(p<0.01)$. Logistic regression analysis didn't suggest inflammatory factor is an important risk factor for anxiety of ACS patients.

Conclusion The study confirmed the western notion that anxiety is a common psychosocial problem, which also applies to Chinese patient s diagnosed with ACS. Anxious ACS patients have rather worse quality of life.

\section{e0284 INTRAVASCULAR ULTRASOUND (IVUS) CHARACTERISTICS OF PREMATURE CORONARY HEART DISEASE (CHD) PATIENTS WITH DIFFERENT RISK FACTORS}

doi:10.1136/hrt.2010.208967.284

Gao Lijian, Chen Jun, Chen Jilin, Yang Yuejin, Qiao Shubin, Xu Bo, Li Jianjun, Qin Xuewen, Yuan Jinqing, Wu Yongjian, Chen Jue, You Shijie, Qian Jie, Dai Jun, Hu Fenghuan, Gao Runlin. Fuwai Hospital

Objectives To observe lesion characteristics of premature coronary heart disease (CHD) patients with different risk factors by intravascular ultrasound (IVUS).

Methods Among 114 premature CHD patients examined by IVUS, quantitative method and qualitative method were adopted to analyse characteristics of coronary artery plaques, and to observe the proportion of different plaques, cross-sectional areas (CSA) surrounded by external elastic membrane, lumen CSA, plaque burden, lumen area stenosis rate and remodelling index of patients with different risk factors.

Results Among the CHD patients with traditional risk factors, there is no statistical difference except in lesion extent and minimum lumen diameter among those $\mathrm{CHD}$ patients with hypertension. The lesions are serious and higher ratio of lipid core to plaque among those CHD patients with diabetes. More soft plaques and mixed plaques are observed among those $\mathrm{CHD}$ patients with hyperlipidaemia. Lesion length and the ratio of lipid core to plaque were significant difference between premature $\mathrm{CHD}$ patients with hyperlipidaemia and control groups. There is no difference on lesion characteristics detected by IVUS between patients with and without family history of CHD. There are more soft plaques and mixed plaques in the premature CHD patients in current smoking group (the $p$ value were $<0.05$ ). The lesions were more serious in current smoking patients than those without smoking patients. There were more ruptured plaques in diabetes group. Positive remodelling is a common phenomenon in diabetes group, while more negative remodelling were observed in other groups.

Conclusions The ratio of lipid core to plaque tends to be higher in group with risk factors than that in group without risk factors. More ruptured plaques were observed among those CHD patients with diabetes. Therefore, these risk factors should be strictly controlled in primary prevention of CHD.

\section{E0285 ANALYSIS OF AETIOLOGY OF IN-PATIENTS FROM THE DEPARTMENT OF HYPERTENSION}

doi:10.1136/hrt.2010.208967.285

Li Nanfang, Wang Lei, Zhou Keming, Wang Xinlin, Zu Feiya, Zhang Delian, Zhang Yanmin, Chang Guijuan. Hypertension Unit of The People's Hospital of Xinjiang Uygur Autonomous Region, The Institute of Hypertension of Xinjiang, The Center of Hypertension of The People's Hospital of Xinjiang Uygur Autonomous Region

Introduction Because some secondary hypertension would be cured by surgery or other special methods, it's important to diagnosis correctly aetiology of hypertension.

Methods Retrospective study was performed based on aetiology of in-patients with hypertension in department of hypertension of Xinjiang from September 1997 to December 2005. 2436 male and 2206 female, with average age was $53.57 \pm 13.14$ were analysed.

Results (1) Of 4642 in-patients with hypertension, there were $85.24 \%$ of essential hypertension and $14.76 \%$ of secondary hypertension (SH). Higher prevalence rate of secondary hypertension were sleep apnoea syndrome (42.92\%) and anxiety (15.04\%). Highest prevalence rate of endocrine hypertension was primary aldosteronism (12.12\%). (2) The male with hypertension was more than the female, the prevalence of essential hypertension (EH), sleep apnoea syndrome (SAS), primary aldosteronism (PA) in male were higher than those of female; the prevalence of anxiety, pheochromocytoma, renovascular hypertension in female were higher than those of male. (3) There was $21.9 \%$ of $\mathrm{SH}$ in youth and $9.85 \%$ of $\mathrm{SH}$ in older.

Conclusion For the youth, SH should be excluded, especially SAS and anxiety. The highest prevalence rate of endocrine hypertension was primary aldosteromsm in youthful and middle aged male. The prevalence rate of pheochromocytoma in female was higher than those of male.

\section{E0286 ANALYSIS OF AETIOLOGY AND METABOLIC DISORDERS OF IN-PATIENTS OF 628 REFRACTORY HYPERTENSION}

doi:10.1136/hrt.2010.208967.286

Wang Lei, Li Nanfang, Zu Feiya, Wang Xinlin, Zhou Keming, Zhang Delian, Chang Guijuan, Zhang Yanmin, Nuer Guli, Zhou Keming, Wang Menghui. Hypertension Unit of The People's Hospital of Xinjiang Uygur Autonomous Region, The Institute of Hypertension of Xinjiang, The Center of Hypertension of The People's Hospital of Xinjiang Uygur Autonomous Region

Introduction Patients with refractory hypertension (RH) were diagnosed by aetiology and treated correctly, which was helpful to control blood pressure and decrease case fatality.

Method Retrospective study was performed based on aetiology and metabolic disorders of 628 in-patients with RH in Department of Hypertension, People's Hospital of Xinjiang, from Sep 1997 to Dec 2005 (361 male and 267 female, average age 55.93 \pm 13.06 years).

Results (1) $80.10 \%$ of 628 in-patients with RH were essential hypertension, $18.95 \%$ of ódcondary hypeòõdnsion $(\mathrm{SH})$ and $0.95 \%$ of indefinite diagnosis. (2) $119 \mathrm{SH}$ comprised $33.61 \%$ of renovascular hypertension, $15.97 \%$ of renal hypertension, $23.53 \%$ of OSAS, $13.45 \%$ of primary aldosteronism, $5.04 \%$ of pheochromocytoma. 
Fewer prevalence of $\mathrm{SH}$ were aortic stenosis (1.68\%), Cushing syndrome $(2.52 \%)$, hyperthyroidism $(0.84 \%)$, and hypothyroidism (3.36\%). (3) There were $66.24 \%$ of dyslipidemia, $43.78 \%$ of abnormality of glucose. Highest prevalence of dyslipidemia was hypertriglyceridemia (41.8\%). Diabetes and IGT were 28.6\%, $15.19 \%$, respectively. (4) The number of overweight and obesity with hypertriglyceridemia and diabetes were significantly higher than those of normal weight.

Conclusion SH should be filtrated in RH. Most RH complicated with Metabolic Disorders, it play an important role on therapy of RH to rectify Metabolic Disorders.

\section{e0287 THE THERAPEUTICAL EFFECT OF THE ADIPOSE DERIVED STEM CELLS}

doi:10.1136/hrt.2010.208967.287

Liu Qian, Wang Ke. Deparment of Cardiology, The First Affiliated Hospital of Dalian

Objective Ischaemic heart diseases, such as acute myocardial infarction, is the most important reason of heart failure. It has been thought that adult cardiac muscle cell is terminal differentiation and would not be regenerated. So now in the clinical, the myocardial ischaemia and other complications cannot be treated thoroughly. Stem cells have got a lot of attention because of the ability of selfrenewal and multi-directional differentiation. Compared with other stem cells, an important clinical advantage of adipose tissue derived stem cells (ADSCs) is that they can be isolated in real time in sufficient quantity with small injuries, so the adipose tissue will have a potential perspective. The objective of this research is to discuss the therapeutical effect of the ADSCs on acute myocardial infarction.

Methods Ligated the left anterior descending coronary artery of the $\mathrm{SD}$ rats to make a model of acute myocardial infarction. The transplantated cells were isolated from the groin and the scapular adipose tissue of the eGFP mice. The ADSCs were transplanted into the infarction area of the experimental group $(n=8)$ by injection but PBS with the same volume as the control group $(n=8)$. Four weeks later, the heart function was measured with echocardiography. The infarction size and the thickness of the ventricular wall were measured by using Masson trichrome stain. All data were expressed as mean \pm SEM. Differences between groups were analysed for statistical significance by the independent sample t-test using SPSS Statistics 11.5. A p-value less than 0.05 denoted a statistically significant difference.

Results Compared with the control group, there were smaller infarction size $(40.0 \pm 8.7 \%$ vs $79.3 \pm 27.1 \%, p<0.01)$, thicker wall $(564.1 \pm 148.1 \mu \mathrm{m}$ vs $189.7 \pm 70.0 \mu \mathrm{m}, \mathrm{p}<0.01)$ in the experimental group as well as the well improved echocardiographic functional parameters, LVESD: $(0.34 \pm 0.17 \mathrm{~cm}$ vs $0.49 \pm 0.25 \mathrm{~cm}, \mathrm{p}<0.01)$, LVEDD: $\quad(0.50 \pm 0.19 \mathrm{~cm}$ vs $0.61 \pm 0.27 \mathrm{~cm}, \quad p<0.05)$, LVFS: $[(31.5 \pm 1.8) \%$ vs $(19.4 \pm 1.4) \%, \mathrm{p}<0.01]$, LVEF: $[(65.6 \pm 3.6) \%$ vs $(45.3 \pm 2.8) \%, p<0.01]$.

Conclusion By injecting into the infarct cardiac muscle, the ADSCs have a positive effect to improve the reconstruction and the systolic and diastolic function in a rat model of acute myocardial infarction.

\section{e0288 ANALYSIS OF THE RATE OF ACHIEVING GOAL BLOOD PRESSURE, BLOOD SUGAR AND BLOOD LIPID IN PATIENT WITH CORONARY ARTERY DISEASE}

doi:10.1136/hrt.2010.208967.288

Li Yan-Fang, Cao Fang-Fang, Cao Xiao-Jing, Shi Shu-Tian. Department of Cardiology, Anzhen Hospital, Capital Medical University, Beijing

Objective To analysis the rates of goal blood pressure, blood glucose and blood lipid achieved in patients with coronary artery disease
(CAD) who were diagnosed by coronary arteriography and to explore the effect of risk factors in $\mathrm{CAD}$.

Methods 2916 cases with CAD were analysed retrospectively the levels of blood pressure, blood sugar and blood lipid when they were admitted to hospital, and the level of blood pressure when they discharged. According to goal values of guidelines, respectively, the effects of three risk factors on $\mathrm{CAD}$ were evaluated. By means of $130 / 80 \mathrm{~mm} \mathrm{Hg}$, the rate of goal blood pressure achieved was $28.4 \%$ $(829 / 2916)$ when they were admitted, and the rate of goal blood pressure achieved was $87.5 \%(2552 / 2916)$ when they were discharged. By means of $140 / 90 \mathrm{~mm} \mathrm{Hg}$, the rate of goal blood pressure achieved was $65.8 \%(1918 / 2916)$ when they were admitted, and the rate of goal blood pressure achieved was $90.0 \%(2624 / 2916)$ when they were discharged. The rate of goal high density lipoprotein cholesterol (HDL-C) achieved, by means of $>1.04 \mathrm{mmol} / \mathrm{l}$, was $34.7 \%(672 / 2916)$ when they were admitted to hospital. The rate of goal low density lipoprotein cholesterol (LDL-C) achieved, by means of $<2.59 \mathrm{mmol} / \mathrm{l}$, was $54.3 \%$ (531/978) in high risk patients, and by means of $<2.07 \mathrm{mmol} / \mathrm{l}$, was $17.4 \%$ (338/1938) in very high risk patients. The rates of goal total cholesterol (TC) achieved, by means of $<4.14 \mathrm{mmol} / \mathrm{l}$, was $49.5 \%$ (484/978) in high risk patients, and by means of $<3.11 \mathrm{mmol} / \mathrm{l}$, was $5.83 \%(113 / 1938)$ in very high risk patients. The rates of goal triglyceride achieved, by means of $<1.7 \mathrm{mmol} / \mathrm{l}$, was $58.0 \%(1690 / 2916)$. There was 538 cases diabetic patients in CAD, occupying $18.5 \%$ (538/2916), diabetic diagnosis standard was fasting blood glucose $>7.0 \mathrm{mmol} / \mathrm{l}$. The rates of goal fasting blood glucose was $1.86 \%(10 / 538)$, by means of $<6.0 \mathrm{mmol} / 1$ fasting blood glucose. There was 600 patients of fasting blood glucose impaired, by means of $>5.6 \mathrm{mmol} / \mathrm{l}$ and $<7.0 \mathrm{mmol} / \mathrm{l}$ on fasting blood glucose, and diabetes together with fasting blood glucose impaired were 1138 cases, occupying 39.03\% (1138/2916) in $\mathrm{CAD}$.

Conclusion The rates of goal blood pressure, fasting blood glucose and blood lipid achieved in patients with CAD were low when they were admitted. Not only rate of goal fasting blood glucose was very low in $\mathrm{CAD}$ accompanying diabetic patients, and but also rates of goal TC and LDL-C achieved were low in CAD. The results show that the management of goal blood pressure, goal fasting blood glucose and goal blood lipid are very important in prehospital for secondary prevention of CAD.

\section{e0289 ASSOCIATION OF LOW ANKLE-BRACHIAL INDEX WITH MORTALITY IN PATIENTS WITH ISCHAEMIC HEART DISEASE}

doi:10.1136/hrt.2010.208967.289

'Zheng Liqiang, ${ }^{2} \mathrm{Li}$ Jue, ${ }^{2} \mathrm{Hu}$ Dayi, ${ }^{3}$ Luo Yingyi, ${ }^{2} \mathrm{Li}$ Xiankai, Xu Yuanxi. ${ }^{1}$ Department of Cardiology, Shengjing Hospital of China Medical University, Shenyang, China; ${ }^{2}$ Heart, Lung and Blood Vessel Center, Tongji University, Shanghai, China; ${ }^{3}$ Shanghai Institute of Health Sciences, Shanghai, China

Objective To find out whether a low ankle-brachial index can improve the prediction of and cardiovascular mortality on top of conventional risk factors remains unclear among patients with ischaemic heart disease. The present study was to assess the association between ankle-brachial index and mortality in Chinese patients.

Methods An observational prospective study was conducted in which 1,800 Chinese patients aged $\geq 35$ years were followed-up from 2004 to 2007-2008.

Results There were 280 deaths, of which 165 were attributable to cardiovascular disease. Compared with patients with an anklebrachial index $\geq 1.1$, the risk of mortality increased linearly in lower ankle-brachial index categories: patients with an ankle-brachial index of 0.9 to $1.1,0.7$ to $0.9,<0.7$ had HR of $1.60,2.07,3.08$ for mortality and 1.89, 2.33, 4.09 for cardiovascular mortality ( $p$ for 